\title{
Dietary sugar, fat and salt intakes in male non-dysvascular lower limb amputees are significantly higher than recommended dietary values in the UK
}

\author{
E.A. Westerkamp, S.C. Strike and M. Patterson \\ Department of Life Sciences, Parkstead House, University of Roehampton, London SW15 4JD
}

Undergoing a lower limb amputation (LLA) is a life-changing experience and the degree to which a patient's health and quality of life is affected depends on several factors including age, cause and level of amputation and co-morbidities (such as cardiovascular disease (CVD), diabetes mellitus or arthritis). ${ }^{(1)}$ Previous research suggests that lower limb amputees are at increased risk of obesity and CVD compared to non-amputees. ${ }^{(2)}$ However, dietary intake, a major determinant of CVD risk, has not previously been studied. The aim of this study was to investigate dietary intakes in adult lower limb amputees.

Dietary intakes of adult males with non-vascular related LLA $(n=46)$ were assessed using a food frequency questionnaire copied from the 'European Prospective Investigation of Cancer' (EPIC) Norfolk study ${ }^{(3)}$ consisting of 130 items commonly consumed in the UK and was based on medium servings or standardised units (i.e. pints, teaspoon, etc.). Results were compared to dietary reference values in the UK published by the Scientific Advisory Committee on Nutrition (SACN) ${ }^{(4)}$ and the Department of Health ${ }^{(5)}$.

The estimated dietary intake risk factors for CVD such as total fat $(34.87 \%)$, saturated fat $(12.72 \%)$, sugars $(22.01 \%)$ and sodium $(2660.10 \mathrm{mg} / \mathrm{d})$ were significantly higher $[p=0.043, p<0.001, p<0.001 ; p<0.001$; respectively] than the dietary reference values.

\begin{tabular}{lccc}
\hline & DRVs & Mean Intakes & Sig. (2-tailed)* \\
\hline Total fat $(\%)^{\dagger}$ & 33 & $34 \cdot 87$ & $\cdot 043$ \\
SFA $(\%)^{\dagger}$ & 10 & $12 \cdot 72$ & $<\cdot 001$ \\
Sugars $(\%)^{\dagger}$ & 10 & $22 \cdot 01$ & $<\cdot 001$ \\
Sodium $(\mathrm{mg})$ & 1600 & $2660 \cdot 10$ & $<\cdot 001$ \\
\hline
\end{tabular}

Values are estimated daily dietary intakes.

DRVs - dietary reference values, SFA - saturated fatty acid

${ }^{*} \mathrm{p}<.05,{ }^{\dagger}$ percent of daily energy intake.

The high intakes of sugars, dietary fats and salts observed in lower limb amputees are concerning. These findings indicate an increased risk of CVD and suggest greater emphasis on dietary intakes should be considered for rehabilitation programmes.

1. Fortington LV, Dijkstra PU, Bosmans JC et al. (2013) J Rehabil Med 45, 587-594.

2. Frugoli BA, Guion KW, Joyner BA et al (2000) JPO 12,80-87.

3. Bingham SA, Gill C, Welch A et al. (1997) Int J Epidemiol 26, S137-51.

4. SACN (2011). Dietary Reference Values for Energy. http://www.sacn.gov.uk/pdfs/sacn_dietary_reference_values_for_energy.pdf (accessed January 2014)

5. Department of Health (1991) Dietary Reference Values for Food Energy and Nutrients for the United Kingdom. Report on Health and Social Subjects 41. The Stationary Office.: HSMO: London. 